\section{Magnitude estimation of apparent sums and differences*}

\author{
WILLIAM E. DAWSON \\ University of Notre Dame, Notre Dame, Indiana 46556
}

Os first scaled two continua by magnitude estimation: apparent area of circles and loudness of $1,000-\mathrm{Hz}$ tones. They then gave magnitude estimations of apparent sums and apparent differences for 15 pairs of stimuli on each of the two continua. The scales for sums and differences were in some cases nearly linearly related to the power function obtained when the same Os scaled the underlying continuum. However, systematic departure from linearity was the usual result. The power law exponents obtained were generally smaller than those usually reported for the two sensory continua.

The past 15 years have seen the birth and rapid growth of a psychophysics based on direct ratio scaling methods (see review by Ekman \& Sjöberg, 1965). A major finding of this endeavor has been Stevens's power law $(1953,1957,1961$ a), which says that psychological magnitude grows as a power function of stimulus intensity. The power law has been shown to hold on more than two dozen sensory continua, and the new methods developed for its demonstration have also been used to quantify attitudes and opinions, cases in which there may be no underlying metric (Stevens, 1966).

The usefulness of the scaling methods raises the question of whether or not they may also be used to describe other psychological operations such as the result of the "mental" combining of two subjective magnitudes from the same sensory continuum. Examples of such mental combinations include subjective addition and subjective subtraction. Such operations may be of the form

$$
\psi_{\text {sum }}=\psi_{1}+\psi_{2}
$$

and

$$
\psi_{\text {diff }}=\psi_{1}-\psi_{2}
$$

where $\psi_{1}$ and $\psi_{2}$ represent any two subjective magnitudes on the continuum and $\psi_{\text {sum }}$ and $\psi_{\text {diff }}$ are their psychological sum and psychological difference, respectively.

According to the power law, $\psi=\mathrm{k} \phi^{\mathrm{a}}$-where $\phi$ refers to stimulus magnitude and $\psi$ refers to the corresponding psychological magnitude.

\footnotetext{
*This research was supported in part by a NIH predoctoral fellowship and was carried out in the Laboratory of Psychophysics, Harvard University. The author is grateful to Bruce Schneider for writing the computer program used in the analysis of the data and for inany helpful discussions of the research.
}

When the appropriate substitutions are made, we obtain:

$$
\psi_{\text {sum }}=k\left(\phi_{1}{ }^{a}+\phi_{2}{ }^{a}\right)
$$

and

$$
\psi_{\mathrm{diff}}=\mathrm{k}\left(\phi_{1}^{\mathrm{a}}-\phi_{2}^{\mathrm{a}}\right)
$$

The purpose of the present experiments was to determine if these equations described direct judgments of sums and differences. The scaling method employed was magnitude estimation.

Results obtained by other investigators suggest that judgments of sums and differences may not prove successful. Torgerson (1960) concludes from his experiments with the lightness of Munsell grays that "It is clear, I think, that magnitude estimations of difference are not at all the same thing as differences computed from the magnitude scale of the attribute itself." Beck and Shaw (1967) find that judgments of difference in loudness are not linearly related to sone scale values. Curtis, Attneave, and Harrington (1968), in a test of a "two-stage model of magnitude judgment," obtain judgments of difference which they fit quite well by an equation that involves two more parameters than Eq. 4. In their approach, the real number continuum is regarded as having a corresponding psychological continuum, with the two not linearly related. Curtis and Fox (1969) have examined analogous equations for the case of subjective sums. On the other hand, Goude (1962) reports that judgments of sums and differences are in accord with his ratio scales for heaviness and visual angle. For heaviness he only examined sums, but for visual angle both sums and differences were found to be in agreement.

The question of whether or not human Os can judge differences on prothetic continua without systematic biases has been raised by Stevens (1961b). According to Stevens, "On prothetic continua it is as though the observer finds himself biased by the fact that a given difference at the low end of the scale is more noticeable and impressive than the same absolute difference at the high end of the scale." Dawson $(1966,1968)$ has attempted to deal with some of the regularities found in such "biased" judgments of apparent difference.

\section{METHOD}

\section{Observers}

The Os were mostly undergraduate students and graduate students, plus a few members of the staff. Approximately the same number of males and females took part in the experiments. Most of the Os took part in at least several of the experiments.

\section{Apparatus}

The circles were drawn with black lines, approximately $5 \mathrm{~mm}$ wide, on $5 \times 7 \mathrm{in}$. cards. The areas of the circles were $4 \pi$, $12 \pi, 36 \pi, 108 \pi, 324 \pi$, and $972 \pi \mathrm{sq} \mathrm{mm}$. The cards containing the circles were placed before the $\mathrm{O}$ on a table at which he was sitting.

The $1,000-\mathrm{Hz}$ tones were produced by an oscillator and were presented binaurally at sound-pressure levels of $40,50,60,70$, 80 , and $90 \mathrm{~dB}$ (re $0.0002 \mathrm{dyne} / \mathrm{cm}^{2}$ ). The PDR-8 earphones were mounted in sponge neoprene cushions MX-41/AR. Two key-operated switches enabled the $O$ to listen to one or the other of two tones, one at a time.

\section{Procedure}

Magnitude estimation was used to obtain the subjective magnitude scales for both apparent area of circles and loudness of 1,000 $\cdot \mathrm{Hz}$ tones. The instructions were modified appropriately for use with judgments of apparent sums and apparent differences. For example, the instructions for judgments of sums were as follows:

"You will be presented with a series of stimuli consisting of pairs of (circles, tones). Your task is to indicate the sum of the apparent (areas, loudnesses) of the two (circles, tones) by assigning a number to the apparent sum for each pair. Assign to the first pair of (circles, tones) any number that seems to you appropriate. Your task will then be to assign successive numbers proportional to your subjective impression of total apparent (area, loudness). Use whatever numbers seem appropriate-fractions, decimals, or whole numbers. For example, if the apparent sum of the first pair of (circles, tones) appears to be 7 times (larger, louder) than the sum of the first pair, assign a number 7 times as large as the first. If it appears $1 / 5$ as (large, loud), give a number $1 / 5$ as large, and so forth." 


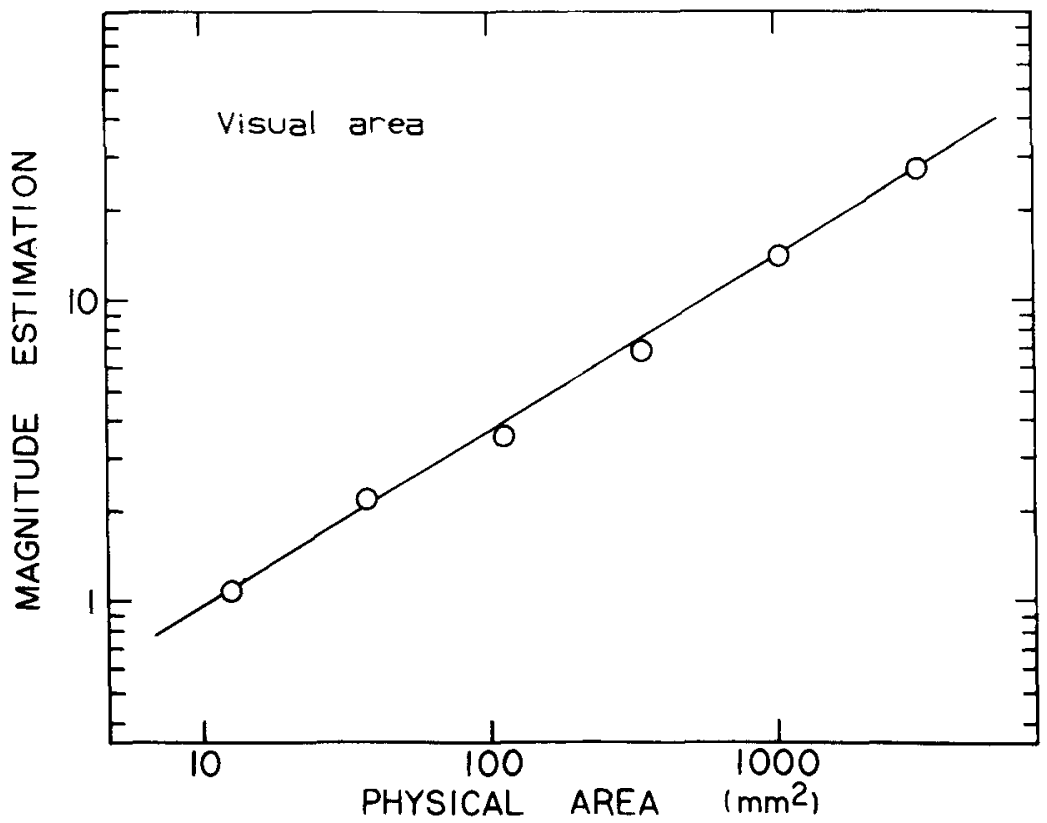

Fig. 1. Apparent area of circles as a function of physical area. In this and all subsequent figures, the points represent geometric means of magnitude estimations.

As a familiarization procedure, the $O$ first judged a series of straight lines that varied in length. The $O$ was instructed to give numbers in proportion to his impression of apparent length.

\section{SUBJECTIVE ADDITION OF VISUAL AREA}

Fifteen undergraduates, who were unpracticed except for an earlier familiarization session, served as Os in two tasks. In the first task, each $O$ made magnitude estimations of the apparent area of the six circles presented three times each in a different irregular order for each $\mathrm{O}$.

In the second task each $O$ gave magnitude estimations of the sum of the apparent areas of pairs of circles. These judgments were to reflect $O$ 's subjective impression of the combined area of each pair of circles. The 15 possible stimulus pairs among the six circles were presented twice each in an irregular order to each $O$.

\section{Results}

Geometric means of the 45 judgments per circle and the 30 judgments per pair of circles were computed. Figure 1 shows the average judgment of apparent area as a function of the physical area of the circle. The straight line in log-log coordinates indicates that apparent area is a power function of physical area. The best-fitting line, obtained by the method of least squares, is described by: $\psi=0.24 \phi^{0.58}$. The exponent, 0.58 , compares witl the value 0.67 obtained with the method of magnitude production by Stevens and Guirao (1963) and the value 0.80 obtained
The data and the line of best fit are plotted in deuble-logarithmic coordinates in Fig. 2. The geometric means of the magnitude estimations have been plotted as a function of the fitted sum $\left(\phi_{1} a+\phi_{2} a\right)$. The line fits the data fairly well, although there appears to be some systematic deviation from a single straight line. It is of interest that the exponent, 0.58 , is the same as that found in the first task.

\section{SUBJECTIVE ADDITION OF LOUDNESS}

The 15 Os who judged area sums, plus one other $O$ who had been famliarized with magnitude estimation, next made similar judgments on the continuum of loudness. In almost all cases, a week had elapsed since the $O$ had completed the area experiments. In the simple magnitude estimation task, each of the six levels of the $1,000-\mathrm{Hz}$ tone was presented three times in a different irregular order to each 0 .

In the second task, the $\mathrm{O}$ gave magnitude estimations of his subjective impression of the sum of the loudnesses of pairs of tones. The 15 possible stimulus pairs among the six tones were presented twice each in a different irregular order to each 0 . The $O$ controlled the presentation of the tones; by pressing one or the other of two keys, one at a time, 0 could listen to the two tones as often and as long as he wished before making his response.

\section{Results}

Figure 3 shows the geometric means of

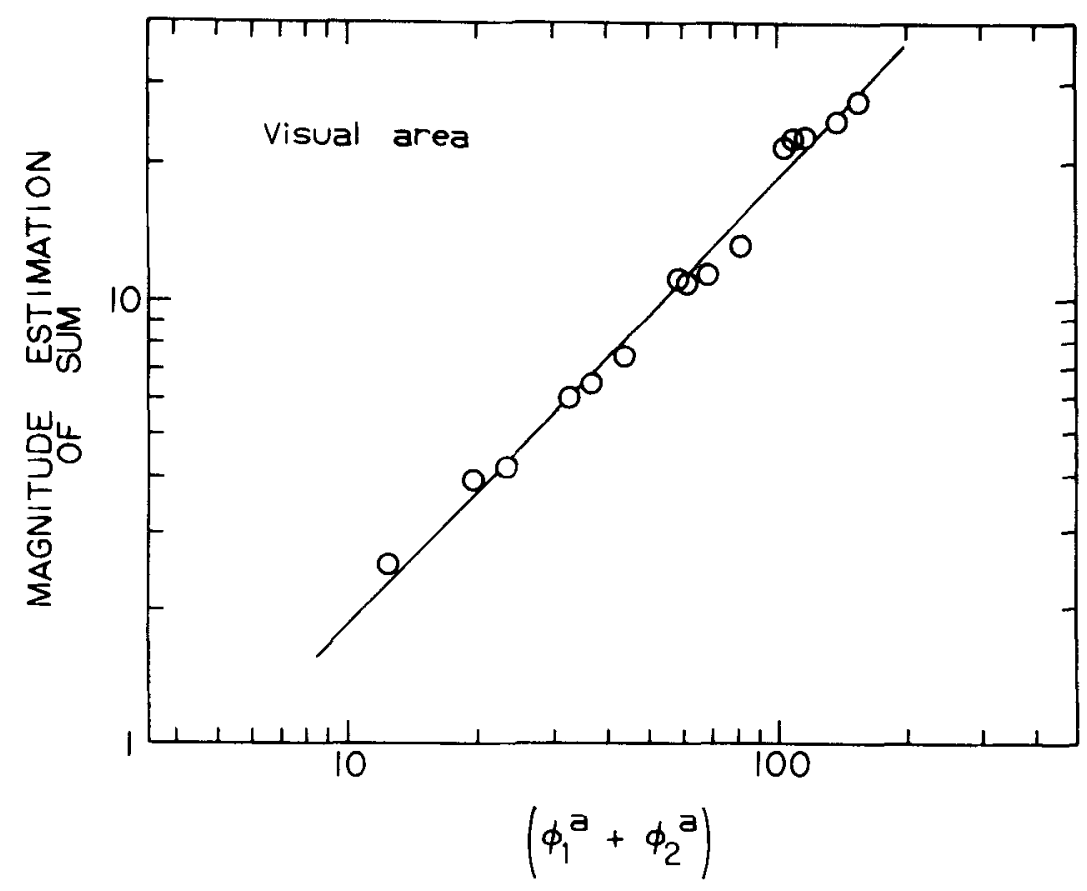

Fig. 2. Apparent sum of pairs of circles as a function of the sum $\left(\phi_{1}{ }^{a}+\phi_{2}{ }^{a}\right)$. 


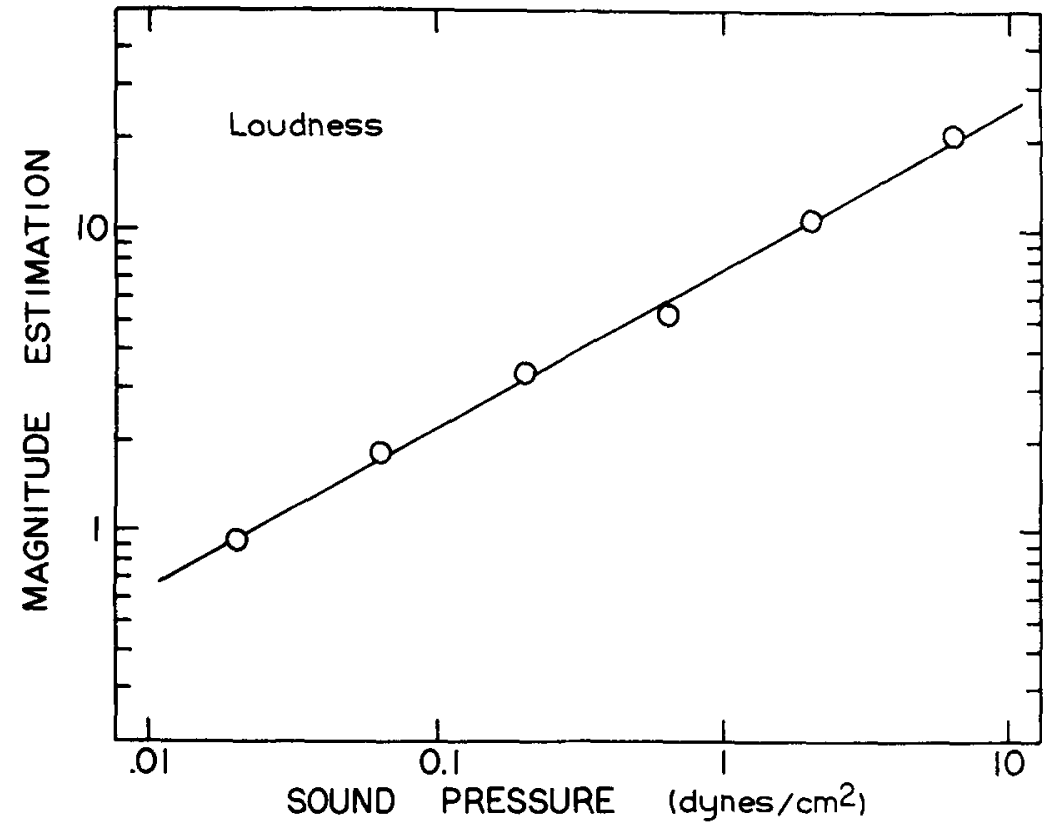

Fig. 3. Loudness of $1,000-\mathrm{Hz}$ tones as a function of sound pressure (dynes/square centimeter).

the judgments as a function of sound pressure (dynes/square centimeter). The line through the data represents the power function: $\psi=7.4 \phi^{0.53}$, where $\phi$ is in terms of sound pressure. This equation, obtained by the method of least squares, has an exponent that is somewhat smaller than the recommended standard value 0.6 (cf. ISO Recommendation R-131; Stevens, 1955). Other Es who obtained smaller exponents for loudness include Hellman and Zwislocki (1961) with 0.54 and Stevens and Tulving (1957) with 0.58 for untrained Os.

The geometric means of the sum judgments were fitted in the same manner as were those for the corresponding area experiment. The iterative procedure gave the following best-fitting equation:

$$
\psi_{\text {sum }}=4.5\left(\phi_{1}^{0.52}+\phi_{2}^{0.52}\right)
$$

Stimulus magnitudes are in terms of sound pressure (dynes/square centimeter). Again the exponent is lower than the standard exponent for loudness, but it is almost equal to the exponent obtained in the preceding magnitude estimation task. In Fig. 4 the geometric means of the sum judgments are plotted as a function of the fitted sums $\left(\phi_{1}^{a}+\phi_{2}{ }^{a}\right)$. The fit is good, but there is a slight curvilinear trend.

\section{SUBJECTIVE DIFFERENCES IN VISUAL AREA AND IN LOUDNESS}

Two groups of 15 graduate students served as Os in two experiments that required judgments of apparent difference. Nine of the Os took part in both of the
In the second experiment, Os were presented with the 15 pairs of tones previously used for the addition task and were asked to give magnitude estimations of the difference in loudness in each pair. Some of the Os asked for a clarification of the word "difference." They were told to judge the subtracted difference in loudness. That is, their numerical estimates should be in proportion to the loudness that remained when the loudness of the softer tone was subtracted from the loudness of the louder one.

\section{Results}

The geometric means of the judgments for each stimulus pair were fitted by an equation of the form $\psi_{\mathrm{diff}}=\mathrm{k}\left(\phi_{1}{ }^{a}-\phi_{2}{ }^{a}\right)$. The best-fitting equations were

Apparent area:

$$
\psi_{\mathrm{diff}}=2.9\left(\phi_{1}^{0.30}-\phi_{2}{ }^{0.30}\right)
$$

Loudness:

$$
\psi_{\mathrm{diff}}=10\left(\phi_{1} 0.58-\phi_{2}{ }^{0.58}\right)
$$

experiments. The second experiment was carried out several weeks after the first. All Os had had previous experience with the method of magnitude estimation.

In the first experiment, Os were shown the same 15 pairs of circles used in the addition task and were asked to give magnitude estimations of the apparent difference in area. At the end of the experiment some Os indicated that the word "difference" was ambiguous (see discussion below).
The exponent for apparent differences in area is small, even smaller than the value found in the experiment on apparent sums. The loudness exponent is fairly close to 0.60 , the value usually found for loudness. Figures 5 and 6 show the average judgments as a function of the fitted differences $\left(\phi_{1}^{a}-\phi_{2}{ }^{a}\right)$. The data in Fig. 5 are fitted poorly by the indicated equation; they depart considerably from the best-fit line and they exhibit an obvious

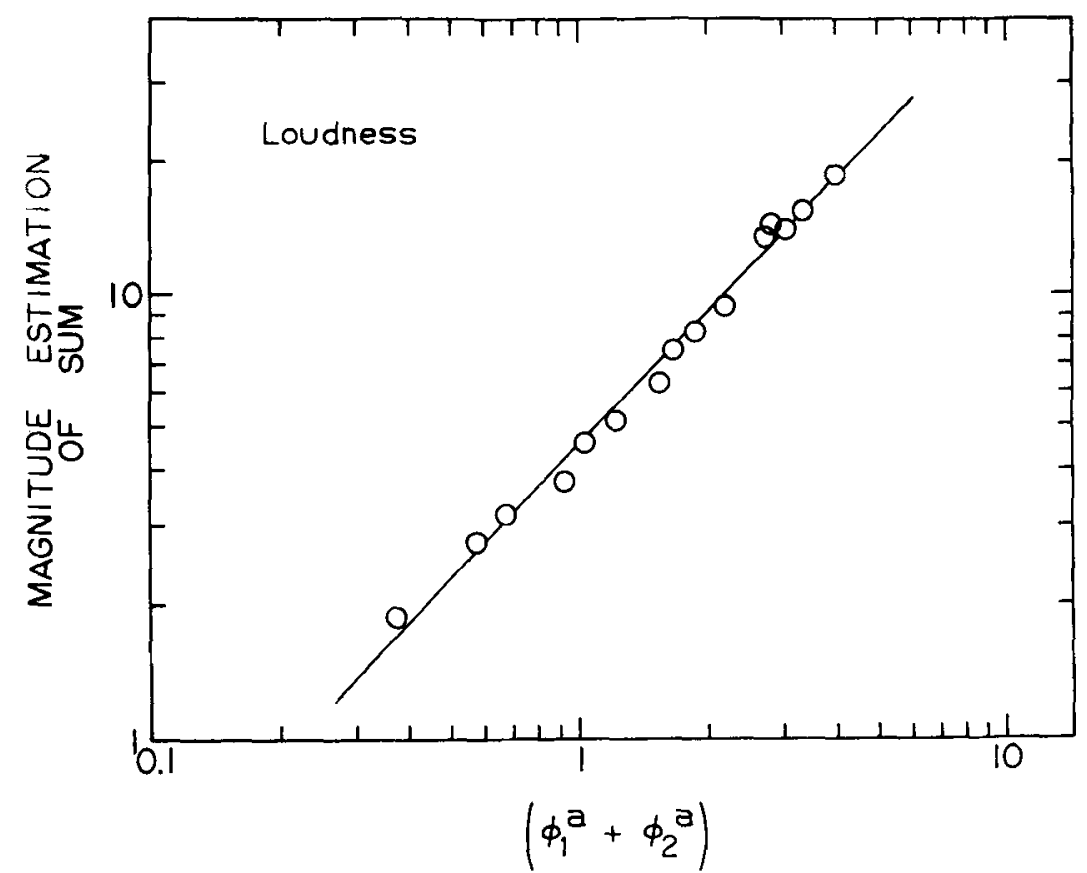

Fig. 4. Apparent sum of pairs of $1,000-\mathrm{Hz}$ tones as a function of the sum $\left(\phi_{1}{ }^{\mathrm{a}}+\phi_{2}{ }^{\mathrm{a}}\right)$. 


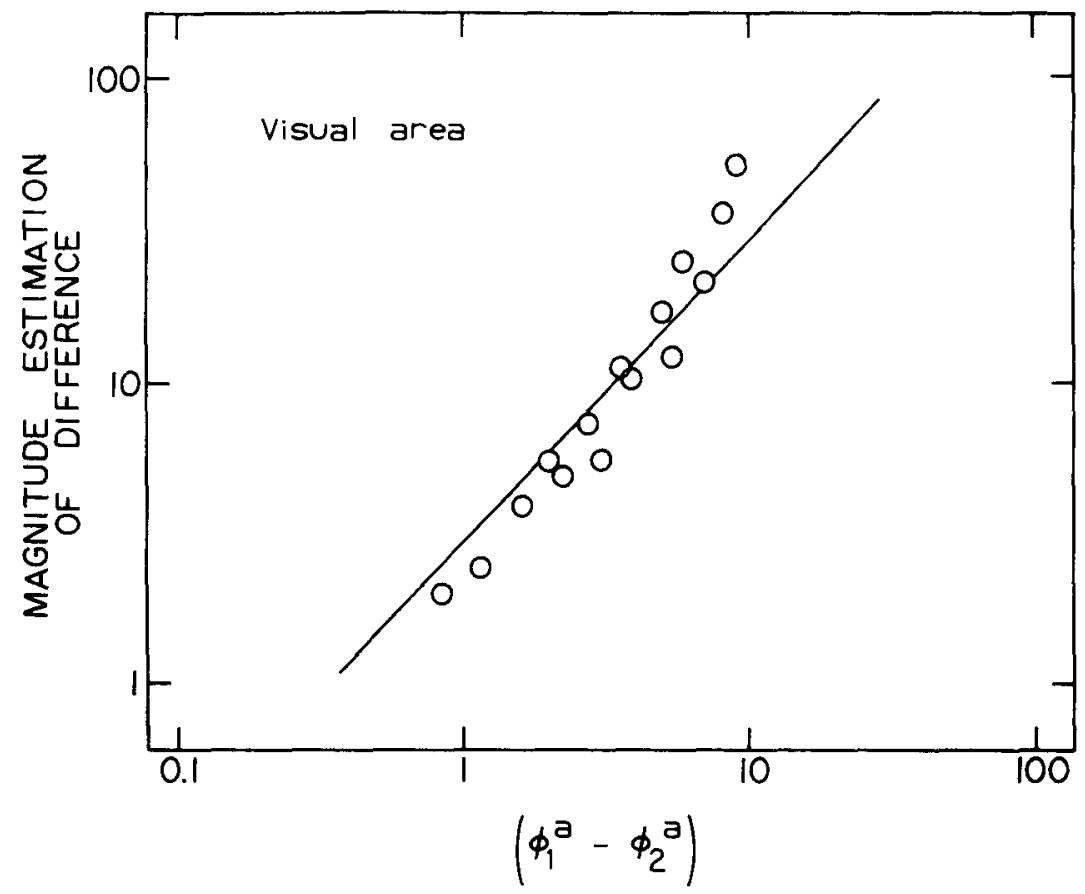

Fig. 5. Apparent difference in visual area of pairs of circles as a function of the difference $\left(\phi_{1}{ }^{a}-\phi_{2}{ }^{a}\right)$.

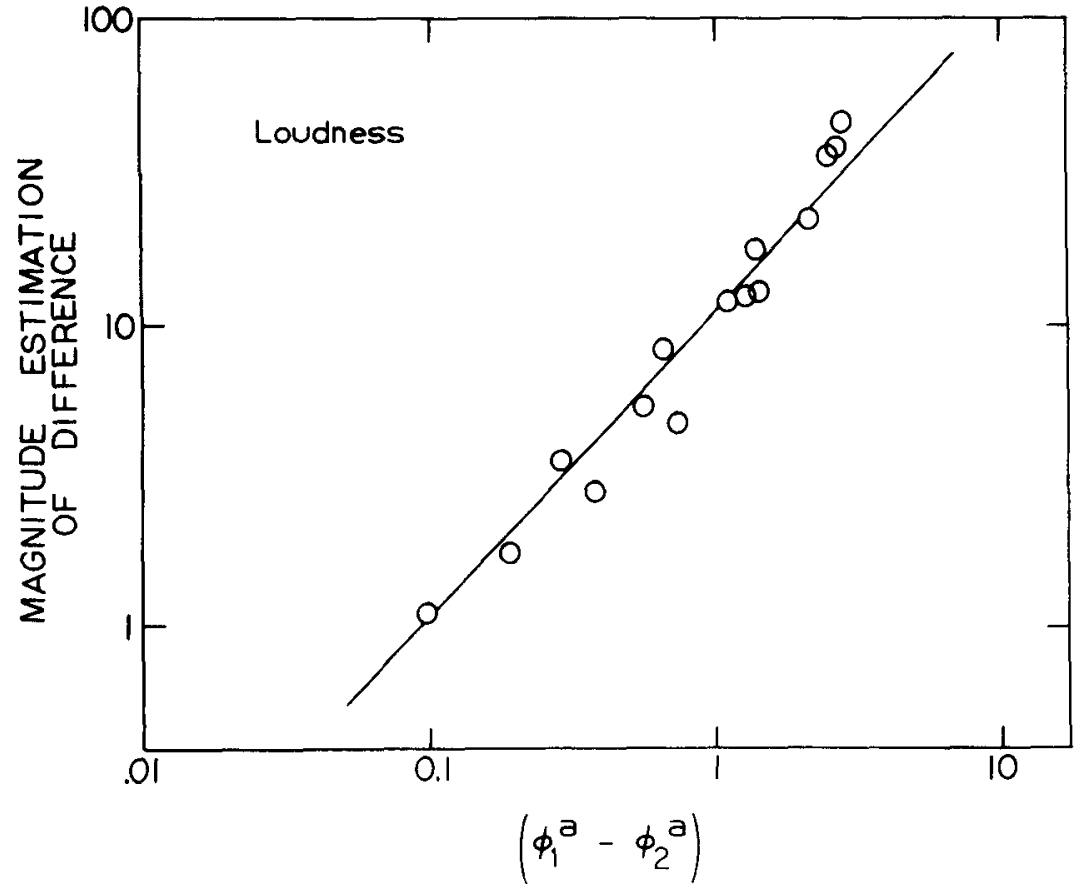

Os said they subtracted their impression of the lesser stimulus from that of the greater and gave magnitude estimations proportional to the remainder. Other Os understood "difference" in the sense of "dissimilarity." These Os reported that they responded to how dissimilar the stimuli in each pair appeared. Some of them indicated that they were influenced by such factors as the apparent ratio between the members of a stimulus pair.

In order to determine if the ambiguity could have affected the results, the two experiments were repeated with two new sets of instructions. One set of instructions asked for magnitude estimations of subtracted difference in apparent magnitude. The other set asked for magnitude estimations of dissimilarity in apparent magnitude.

The Os for the four tasks were for the most part the same as those who served in the experiment on addition of loudness. The same 16 Os now took part in the two experiments on subtracted difference. Two weeks later, 14 of the 16 plus one other inexperienced $O$ took part in the two dissimilarity experiments.

\section{Results}

The data from each of the four experiments were analyzed as before, and the parameters of the best-fitting equations are given in Table 1, along with those of the other experiments.

The area experiment that involved subtracted differences yielded an exponent of 0.55 , a value close to the exponent 0.58 obtained with most of the same Os in the magnitude and the addition experiments for apparent area. On the other hand, these Os gave an exponent of 0.33 when asked to judge the dissimilarity of apparent area. This value is close to the 0.30 obtained with the graduate students as Os.

The loudness results did not give different exponents for the two types of instruction, namely subtracted difference and dissimilarity. Both instructions yielded 0.46 as the exponent, a value smaller than the 0.58 obtained from the judgments of difference by the graduate students.

Fig. 6. Apparent difference in loudness of pairs of tones as a function of the difference $\left(\phi_{1}{ }^{a}-\phi_{2}{ }^{a}\right)$.

Table 1

curvilinearity. The data of Fig. 6 show less curvature, but they vary considerably about the line of best fit.

\section{AMBIGUITY OF "DIFFERENCE"}

As noted above, some of the Os seemed uncertain about what it was that they were supposed to do in judging differences. Their comments made it clear that the word "difference" was ambiguous. Some

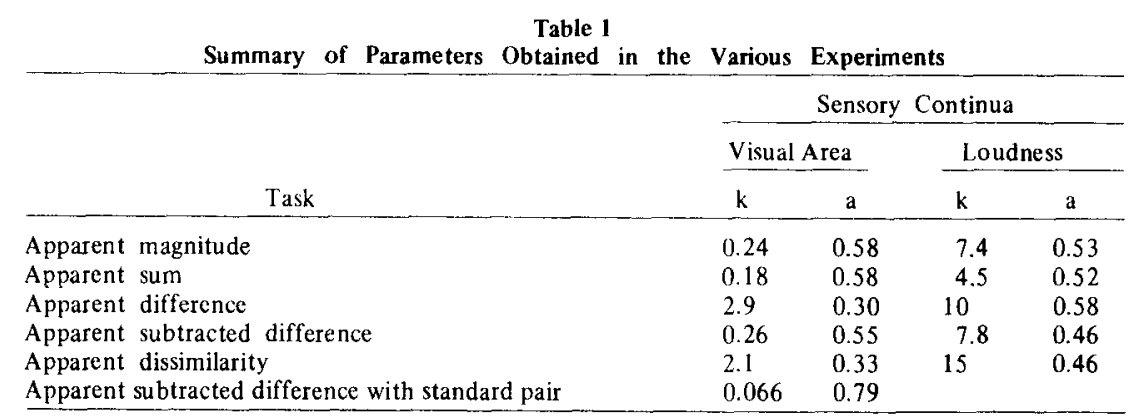




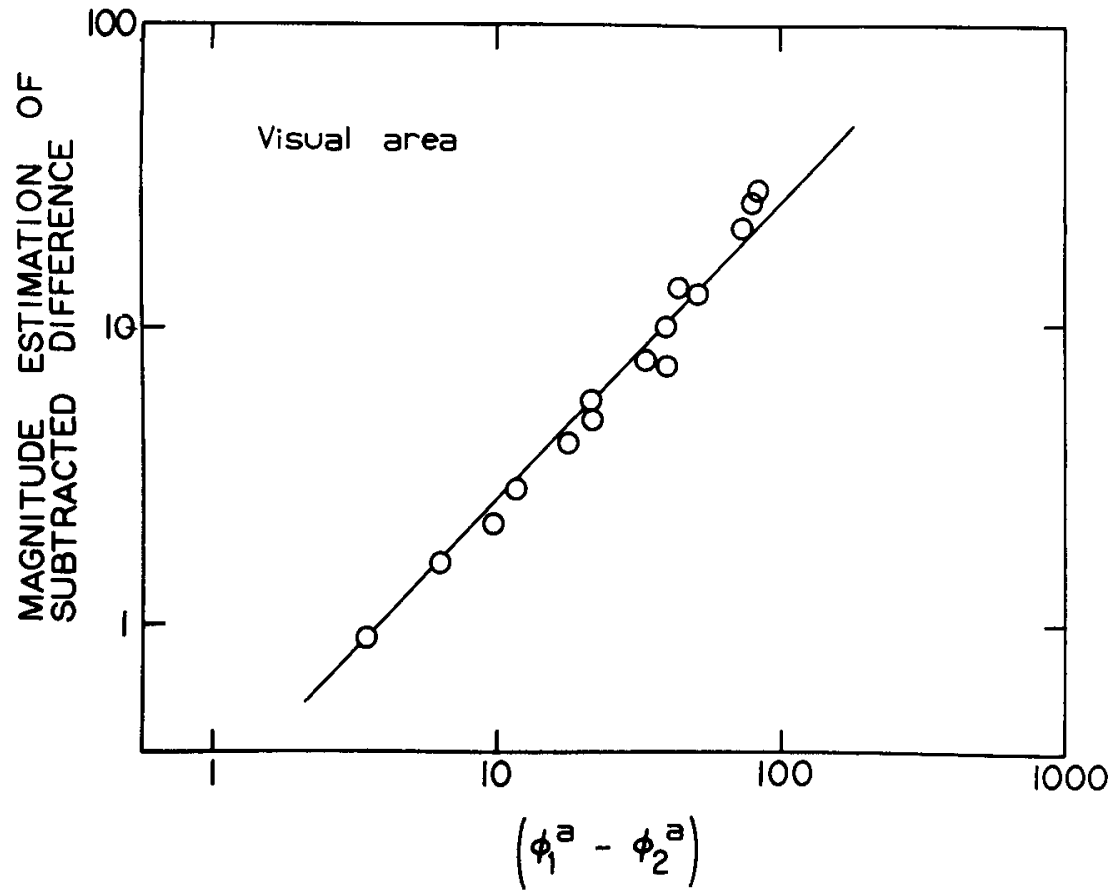

Fig. 7. Apparent subtracted difference in visual area of pairs of circles as a function of the difference $\left(\phi_{1}{ }^{a}-\phi_{2}{ }^{a}\right)$.

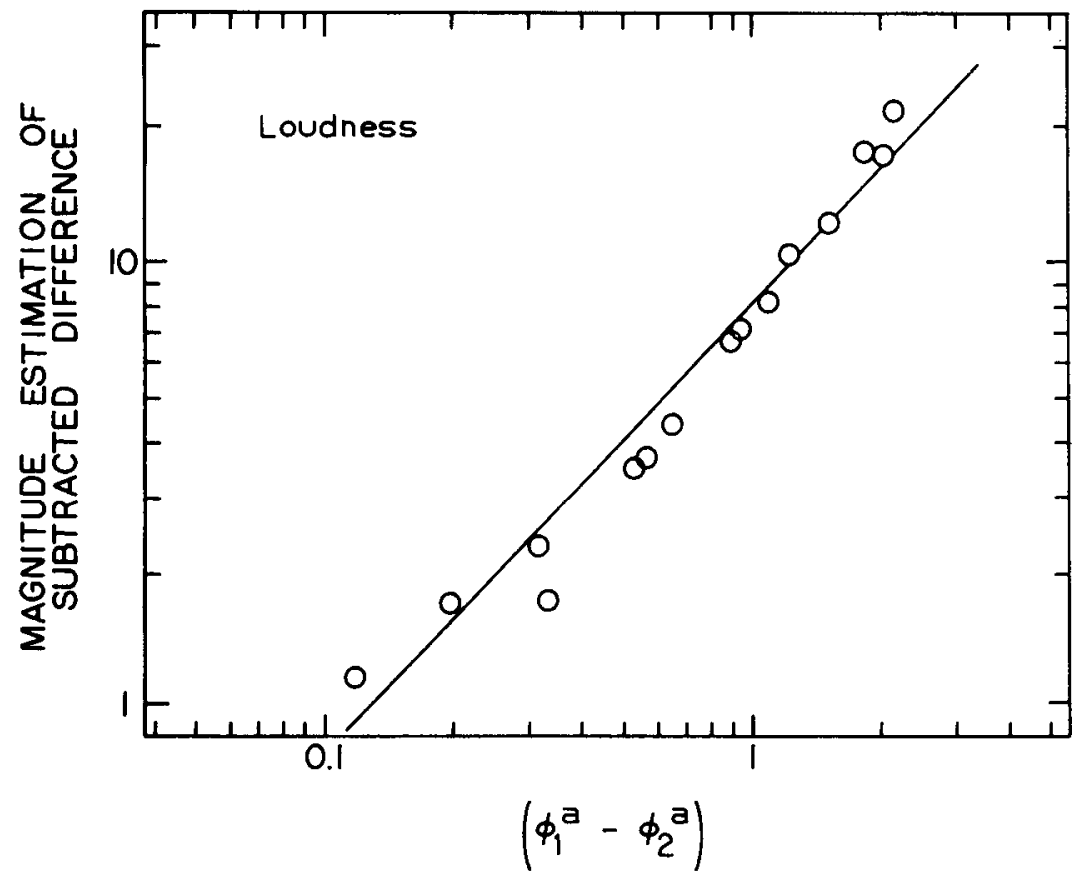

Fig. 8. Apparent subtracted difference in loudness of pairs of tones as a function of the difference $\left(\phi_{1}{ }^{a}-\phi_{2}{ }^{a}\right)$.

Figures 7 through 10 show the results of the four different experiments. All show considerable variation about the fitted lines. Figure 7, which involved subtracted differences in apparent area, seems to show the best fit. There is at least a hint of curvilinearity in all these figures, and Fig. 9 is especially curved, much like Fig. 5. linear, but some systematic deviation seems present. Also, the line of best fit departs from unit slope. The difference judgments are much more scattered and are not fitted well by a straight line. Similar plots for the other experiments where Os judged difference (or dissimilarity) in loudness show a comparable amount of scatter.

\section{SUBTRACTION IN THE PRESENCE OF A STANDARD PAIR}

An experiment on the subtraction of areas was carried out in which the first pair of circles was left visible in front of the 0 while he judged each of the remaining pairs. The purpose was to see if a decreased memory burden might give more orderly data.

Eight graduate students and two faculty members served as Os. All were experienced with the method of magnitude estimation. Three had served in the first two difference tasks. The same 15 pairs of circles were used. Instructions were of the subtracted-difference variety and were modified only slightly to refer to the continued presence of the first pair of circles.

\section{Results}

The geometric means of the judgments were treated as in the other difference experiments. The parameters of the best-fitting equation are given in Table 1 . The value for the exponent, 0.79 , is within the range of exponents usually reported for apparent area. Figure 12 shows that the data are fairly well fitted by a model based on subjective differences, although there is some systematic variation about the best-fitting line.

\section{DISCUSSION}

In some of the experiments reported, judgments of apparent sum and apparent difference came close to being describable by equations of addition and subtraction derivable from Stevens's power law. The addition of apparent area and of loudness gave results that were nearly linearly related to their corresponding magnitude scales. That is, the exponents for the ordinary magnitude scale and for the sensory addition results were almost identical. The data, however, show some systematic departure from the best-fitting lines. Also, the sizes of exponents obtained are smaller than those usually reported for these two sensory continua.

For both area and loudness, subtraction results agree less well with their corresponding magnitude scales than do the addition results. The ambiguity of the word "difference" may be the cause of some of the discrepancy. With visual area, the exponent for the judgment of 


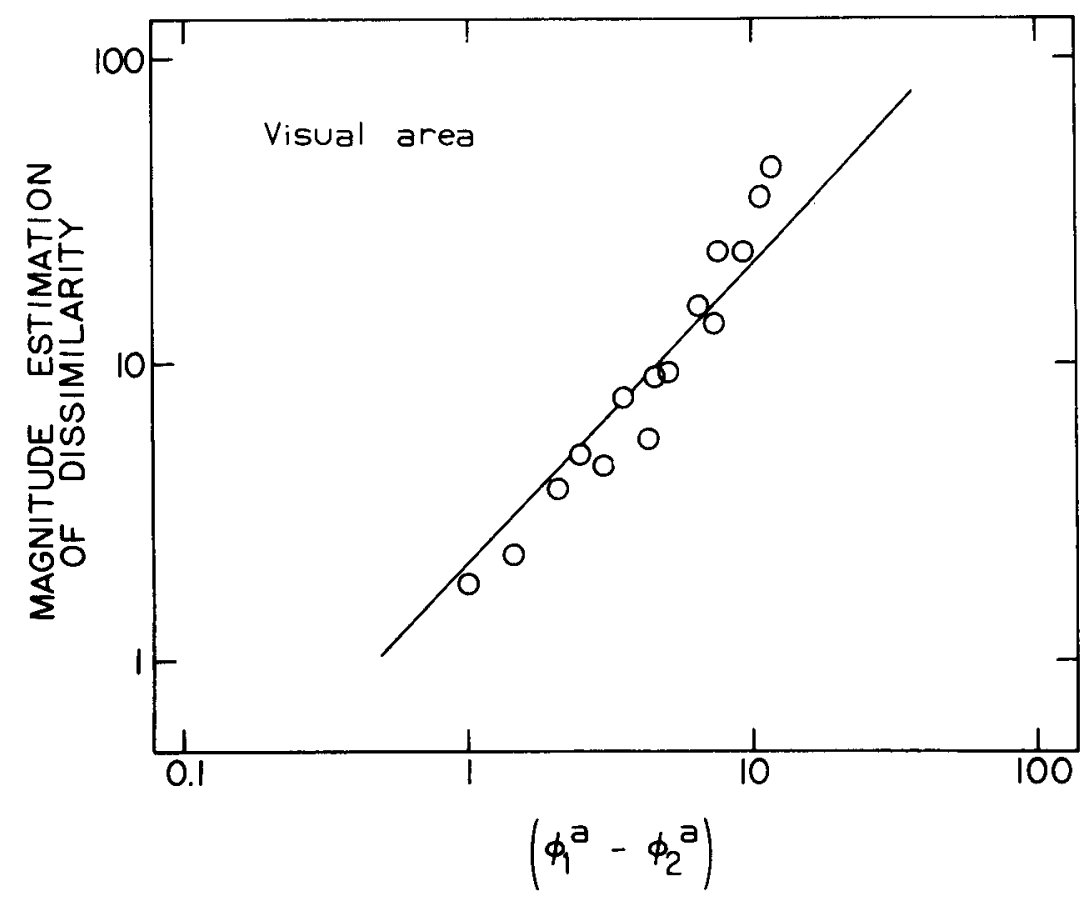

Fig. 9. Apparent dissimilarity in visual area of pairs of circles as a function of the difference $\left(\phi_{1}{ }^{a}-\phi_{2}{ }^{a}\right)$.

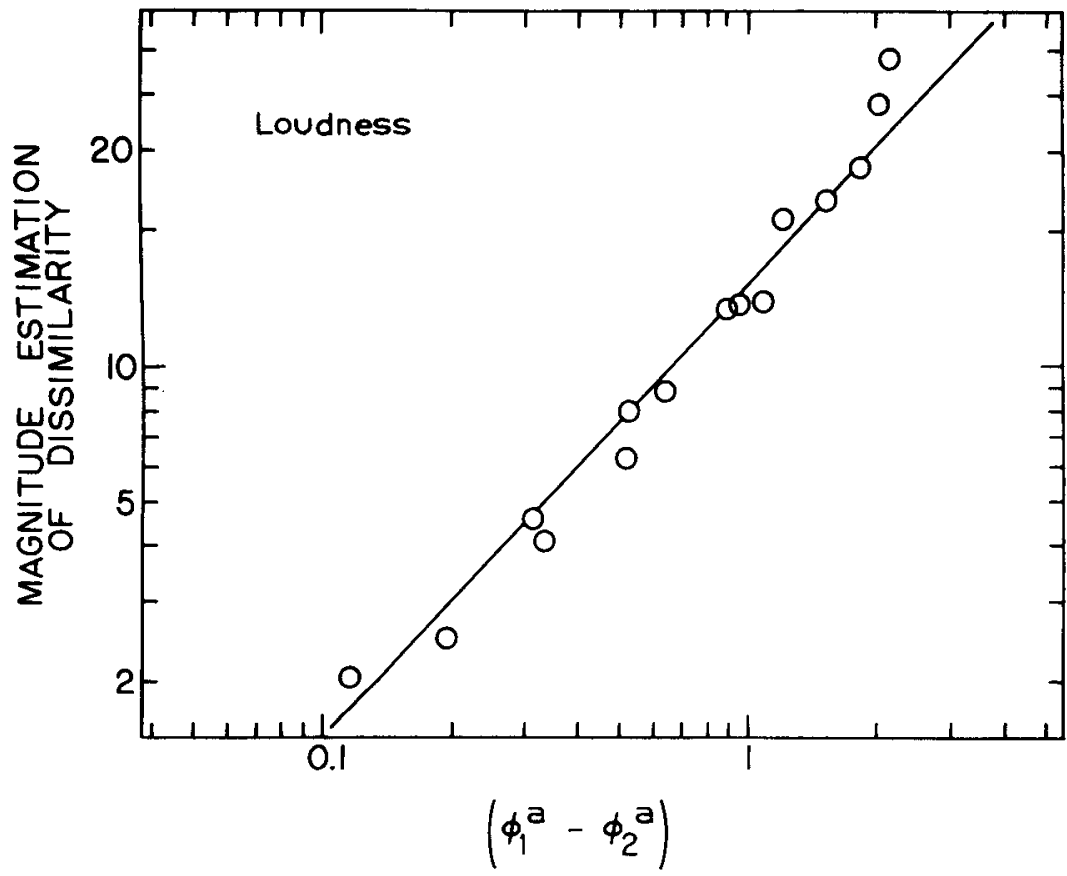

Fig. 10. Apparent dissimilarity in loudness of pairs of tones as a function of the difference $\left(\phi_{1} \mathbf{a}-\phi_{2} \mathbf{a}\right)$.

subtraction is only slightly smaller than that for the obtained magnitude scale. The final experiment, which employed a visible standard pair of circles, yielded the results that accord best with the usual magnitude scale for apparent area.

The loudness results secm to vary considerably about the subtraction equation derived from the power law. Differential effects due to different instructions are not so clear-cut with loudness as they are with apparent area. In both difference tasks that employed relativcly inexperienced $\mathrm{Os}$, the exponent is clearly less than that of the obtained magnitude scale for loudness. On the other hand, the exponent for the difference task that employed graduate students is close to the standard value. It is possible that some of these Os had previously judged

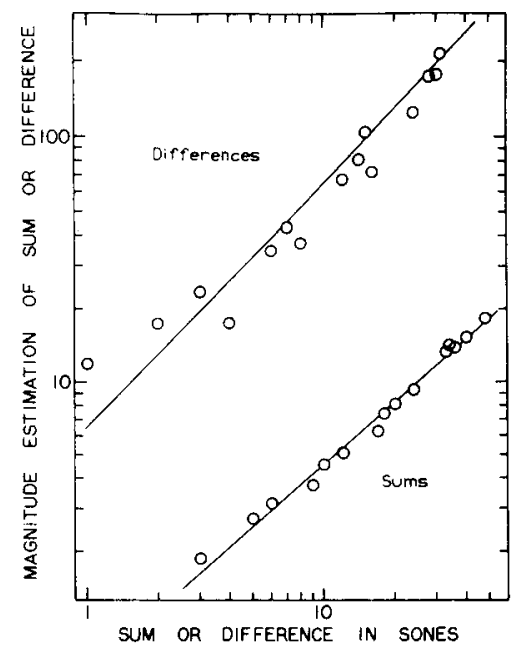

Fig. 11. Apparent sum and the apparent subtracted difference in loudness as a function of the sum and the difference in sones, respectively. For display, the difference data have been shifted upward by one log unit.

"dissimilarity" in the area experiment and that they changed over to judging "subtracted difference" in the loudness task as a result of the discussion with the $\mathrm{E}$ following the area experiment. As mentioned, some Os indicated at that time that they thought the instructions ambiguous.

The various factors that appear to have affected the experiments are worth summarizing. The present sample of inexperienced Os seems to have given data for which exponents are smaller than those obtained with groups of experienced Os. Instructions to judge subtracted difference appear to give results in closer agreement with the corresponding magnitude scale than do vague instructions to judge difference. The memory burden of the $O$ would seem to be greater for loudness judgments than for apparent area. The two circles of each pair were presented simultaneously, whereas the two tones of each pair were presented successively. Consequently, loudness data might be expected to be noisier than data for apparent area.

Although the results for subjective addition and subtraction are in some cases nearly linearly related to their simple magnitude scales, there clearly remains systematic deviation about the lines of best fit. The nature of these deviations requires further investigation. They do not seem to be due to the two-stage process suggested by Curtis et al (1968). unless it is assumed that the exponent for the "sensory continuum of numbers" is fairly close to unity. For example, the fit in Fig. 4 can be improved upon if the number continuum is 


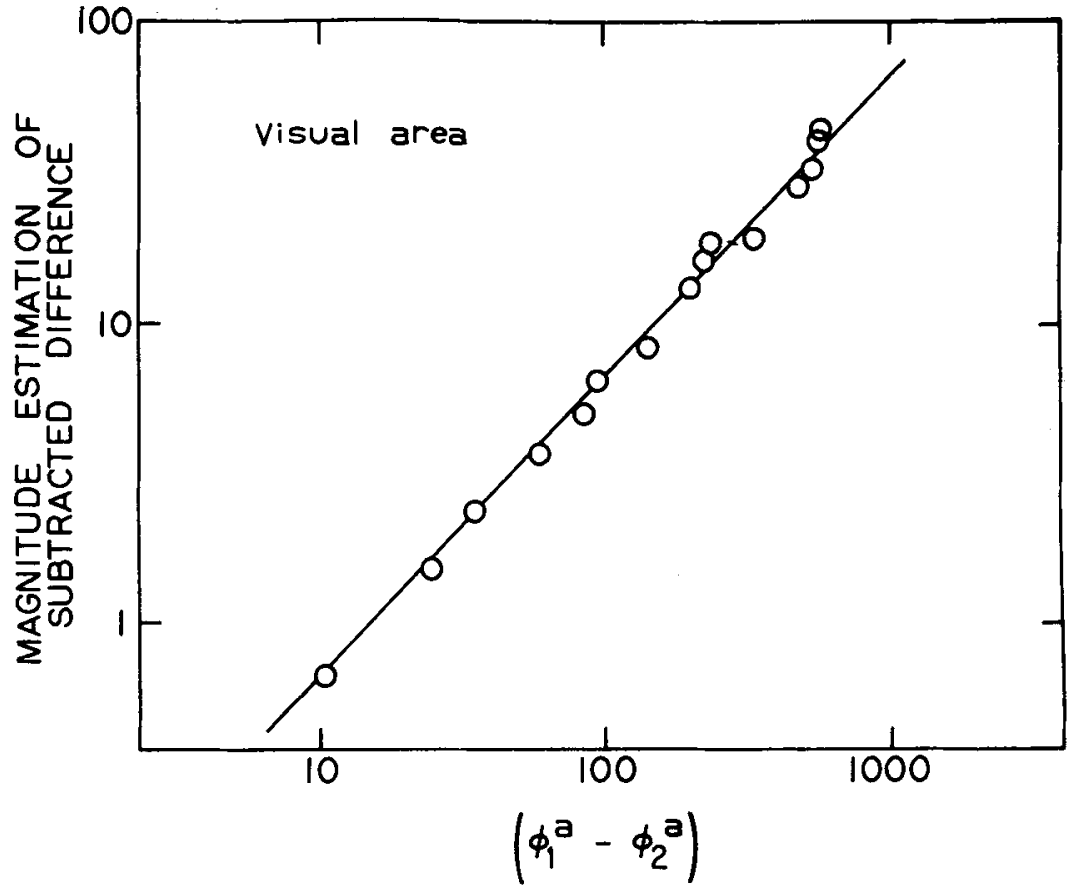

Fig. 12. Apparent subtracted difference in visual area of pairs of circles as a function of the difference $\left(\phi_{1} a-\phi_{2} a\right)$. In this experiment, the first pair of stimuli was left in front of the $O$ while he judged each of the remaining pairs.

taken as having an exponent of 0.94 and that in Fig. 12 if it has 1.2 as exponent. As Curtis et al (1968) report, Attneave "inferred that the exponent for numbers would be of the order of 0.40 .'

For the present, it must be concluded that magnitude estimation of apparent sums and differences yields results that are not in strict accord with the basic sensory scales obtained by magnitude estimation of individual stimuli from the same continuum. Thus, ratio scales of apparent psychophysical judgments. Perception \& Psychophysics, 1969, 5, 89-93.

DAWSON, W. E. Direct scaling of stimulus differences. Paper presented at meeting of the Eastern Psychological Association, New York, April 1966.

DAWSON, W. E. An experimental analysis of judgments of sensory difference. Unpublished doctoral thesis, Harvard University, 1968.

EKMAN, G., \& SJÖBERG, L. Scaling. Annual Review of Psychology, 1965, 16, 451474.

GOUDE, G. On fundamental measurement in psychology. Stockholm: Almqvist \& Wiksell, 1962.

HELLMAN, R. P., \& ZWISLOCKI, J. Some factors affecting the estimation of loudness. Journal of the Acoustical Society of America, 1961, 33, 687-694.

STEVENS, J. C. \& TULVING, E. Estimations of loudness by a group of untrained observers. American Journal of Psychology, 1957, 70, 600-605.

STEVENS, S. S. On the brightness of lights and the loudness of sounds. Science, 1953,118, 576.

STEVENS, S. S. The measurement of loudness. Journal of the Acoustical Society of America, $1955,27,815-829$.

STEVENS, S. S. On the psychophysical law, Psychological Review, 1957, 64, 153-181.

STEVENS, S. S. The psychophysics of sensory function. In W. A. Rosenblith (Ed.), Sensory communication. New York: Wiley, 1961a.

STEVENS, S. S. Toward a resolution of the Fechner-Thurstone legacy. Psychometrika, 1961b, 26, 35-47.

STEVENS, S. S. A metric for the social consensus. Science, 1966, 151,530-541.

STEVENS, S. S., \& GUIRAO, M. Subjective scaling of length and area and the matching of length to loudness and brightness. Journal of Experimental Psychology, 1963,66, 177-186.

TEGHTSOONIAN, M. The judgment of size. American Journal of Psychology, 1965, 78, 392-402.

TORGERSON, W. S. Distances and ratios in psychophysical scaling. M.I.T. Lincoln Laboratory Report 58-G-0014, October 1960. HARRINGTON, T. L. A test of a two-stage
model of magnitude judgment. Perception \& Psychophysics, 1968, 3, 25-31.

CURTIS, D. W., \& FOX, B. E. Direct quantitative judgments of sums and a two-stage model for 